\title{
Design a Wireless Meteorological Station in Jordan
}

\author{
Yahya S. H. Khraisat \\ Electrical and Electronics Department \\ Al-Huson University College, Al-Balqa' AppliedUniversity \\ PO box 50, Al-Huson 21510, Jordan \\ E-mail: yahya@huson.edu.jo
}

Received: September 19, 2011

Accepted: November 21, 2011

Published: January 1, 2012

doi:10.5539/cis.v5n1p87

URL: http://dx.doi.org/10.5539/cis.v5n1p87

\begin{abstract}
This paper demonstrates a low-cost, yet reliable, weather monitoring system. The proposed system has three sensors that measure the temperature, wind speed and wind direction, respectively. The analogue outputs of the sensors converted to digital signals and further processed by a microcontroller. Using easily-available components and simple circuitry, the system should be beneficial in providing a portable and low-cost weather monitoring using wireless communication system. Until now these parameters in the meteorological department in Jordan are transmitted by telephone. In this system we developed an automated system which continuously measures weather parameter and transmit them to the main meteorological station. In future we are planning to have more substations which can interact with main station.
\end{abstract}

Keywords: Transmitter, Receiver, Temperature Sensor, Wind Sensors, Microcontroller, Antenna

\section{Introduction}

The meteorology system is a PIC-based (16F877A) project designed to take readings from temperature, wind speed and wind direction sensors. All of the readings are taken to the PIC in transmitting station and displayed on a $16 \times 2 \mathrm{LCD}$ then they are transmitted to receiving station, where the reading displayed on $16 \times 2 \mathrm{LCD}$. Basically a meteorological system consists of transmitting and receiving stations.

\subsection{System Objectives and Operation}

System objectives:

(1) Building one part of the weather station (transmitting station), to measure weather parameters, such as temperature, wind speed and wind direction. The project involves using microcontrollers (PIC), encoder and ASK transmitters for sending data to a receiving station.

(2) Investigating a complete weather station that transmits radio signals such as environmental temperature, wind speed and wind direction, to the receiver station.

System operation:

To monitor and display the temperature, wind speed and wind direction of the atmosphere, using analogue and digital components, the analogue outputs of the sensors are connected to a microcontroller through an ADC. An LCD display is also connected to the microcontroller to display the measurements and to transmit them to the receiving station.

\subsubsection{System Components}

- 2 Microcontrollers (PIC16F877A)

-Sensors (wind vane, wind cup and LM 35)

- CIP-8 Encoder/Decoder

- $433 \mathrm{MHz}$ Tx/Rx

- 2 LCD $16 * 2$

-2 Operational Amplifiers

- 2 Antennas 
-DC generator

- Wiper potentiometer

\subsection{Main Block Diagram}

The meteorological system consists of two main parts: the transmitting station and the receiving station. The transmitting and receiving stations shown in Figures 1 and 2, consist of many functional sections: the PIC16F877 microcontroller, antenna, LCD and the power supply. In transmitting station there are 3 sensors, ASK transmitter and CIP-8 Encoder while in the receiving station there are ASK receiver and CIP-8 Decoder.

\section{Sensors}

\subsection{Definition}

A sensor is a device that receives a signal and responds with an electrical signal, while transducer is a converter of one type into another. In practice, however the terms are often used interchangeably. Sensors are used to measure various physical proprieties such as temperature, force, pressure, etc. These proprieties act as stimulus to the sensor and the sensor output is conditioned and processed to the corresponding measurement of the physical property.

\subsection{Sensor Types}

\subsubsection{Temperature Sensor}

Temperature is measure of how fast the particles of a substance are moving. Temperature was one of the easiest parameters to measure (Christopher, 2005). A sensor used to measure the current external temperature, and return this value to microcontroller (PIC16F877A). We used integrated circuit temperature sensors with various configurations. A common example is the LM34 and LM 35 series. The LM 35 produces an output that is proportional to Celsius temperature. The temperature is sensed using an LM35 temperature sensor chip (see Figure 3). Notice that it has three active terminals: supply voltage (VS), ground and output voltage (Vout). This chip produces a signal of $10 \mathrm{mV}$ per degree Celsius. The signal of $10 \mathrm{mV}$ however is too small to be detectable by the microcontroller. We employed a TL071cp Op-Amplifier chip (see Figure 4), to amplify the voltage output of the LM35, and this output is sent to the microcontroller (see Figure 8).

\subsubsection{Wind Speed Sensor}

Wind is a vector quantity and it is more common to consider wind speed (the magnitude of the vector). Cup-anemometer has been used to sense wind velocity. An anemometer is useful because it rotates with the wind. The cup anemometer usually consists of three conical or spherical cups mounted on a rotating shaft (see Figure 5). The speed of rotation is proportional to wind speed (Gary).

Almost any design of cup anemometer will work; an optimum design will include the following features:

The rotating part of the anemometer should be as light as possible to start at low wind velocities and respond quickly to wind gusts. The anemometer should have three cups. Two cups won't start rotation reliably and more than three cups simply add the mass of the rotating part.

The cups should ideally have a conical shape but spherical is also acceptable. The edges of the cups should preferably be beaded (rounded) to reduce turbulence. A DC generator (or DC motor driven as a generator) generates a terminal voltage proportional to its rotational speed (see Figure 6), so it is useful in generating an electrical voltage proportional to wind speed, and then connected to operational-amplifier which amplifies the output signal from DC generator. Then the output of the operational amplifier will be connected to the microcontroller.

\subsubsection{Wind Direction Sensor}

Wind direction is defined as the direction, which the wind is blowing from. A wind vane is also called a weather vane; it is a tool for measuring wind direction. As the wind blows from one direction, the wind vane adjusts itself to point in the wind direction. The position of the pointer is interpreted by a transducer, which produces an electrical signal relative to a position. Normally a potentiometer is used as the transducer. The wind vane consists of an arrow shaped wind vane (made of cardboard) attached to rotary potentiometer (see Figure 7). This type of weather vane has the rotating shaft connected to a variable resistor. The electronic signal output from a wind vane derives; a wiper potentiometer (rotary potentiometer). The variable resistor produces a variable voltage depending on the resistance value. This voltage can be connected to an ADC in the microcontroller. The design of the three sensors is shown in sensor box (see Figure 11). 


\section{Microcontroller}

A microcontroller is a computer-on-a-chip, or, a single-chip computer. Micro suggests that the device is small, and controller tells you that the device might be used to control objects, processes, or events. Another term to describe a microcontroller is embedded controller, because the microcontroller and its support circuits are often built into, or embedded in the devices they control. In contrast, a microcontroller is a single-chip computer because it contains memory and I/O Interfaces in addition to the CPU. The Main parts of a microcontroller are:

(1) Central Processing Unit (CPU).

(2) Random Access Memory (RAM

(3) Read Only Memory (ROM).

(4) Input/output lines (I/O lines).

(5) Timers and other peripherals such as an analog to digital (A/D) and digital to analogue (D/A) converters.

Why we used a Microcontroller?

The microcontroller's ability to store and run unique programs makes it fairly flexible. For example, one can program a microcontroller to perform functions based on predetermined situations (I/O-line logic) and selections. The microcontroller's capability to carry out mathematical and logical functions allows it to imitate complicated logic and electronic circuits. As we said microcontrollers are very efficient at processing digital numbers, so an analog-to-digital converter used to convert an analog voltage level to a digital number. The microcontroller can then efficiently process the digital representation of the original analog voltage.

\subsection{PIC Microcontroller}

PIC is a family of RISC microcontroller. It is generally regarded that PIC stands for: 1- "Peripheral Interface Controller" 2- "Programmable Intelligent Computer". The PIC 16F877 microcontroller is chosen because it is low cost, reliable, easy to use and capable of performing a wide range of tasks. The required tasks by the proposed system are carried out via software using microbasic language. And in the fact it consumes very little DC power and it has embedded within the device.

\subsection{Encoder}

An encoder is a device used to change a signal (such as a bit stream) or data into a code. The code may serve any of a number of purposes such as compressing information for transmission or storage, encrypting or adding redundancies to the input code, or translating from one code to another. This is usually done by means of a programmed algorithm, especially if any part is digital, while most analog encoding is done with analog circuitry. We used in our project CIP-8 Encoder (see Figure 12).

\subsection{ASK Transmitter}

A transmitter is an electronic device which usually with the aid of an antenna propagates an electromagnetic signal such as radio, television or other telecommunications. Amplitude-shift keying (ASK) is a form of modulation that represents digital data as variations in the amplitude of a carrier wave. The amplitude of an analog carrier signal varies in accordance with the bit stream (modulating signal), keeping frequency and phase constant. The level of amplitude can be used to represent binary logic 0 s and $1 \mathrm{~s}$. One binary digit represented by presence of carrier, at constant amplitude and other binary digit represented by absence of carrier. We can think of a carrier signal as an ON or OFF switch. In the modulated signal, logic 0 is represented by the absence of a carrier thus giving OFF/ON keying operation and hence the name given. Amplitude Shift Keying was the particular scheme chosen because it is also relatively easy to implement and relatively inexpensive.

\subsection{Decoder}

A decoder is a device which does the reverse of an encoder, undoing the encoding so that the original information can be retrieved code. The same method used to encode is usually just reversed in order to decode. In digital electronics this would mean that a decoder is a multiple-input, multiple-output logic circuit that converts coded inputs into coded outputs, where the input and output codes are different. We used in our project CIP-8 Decoder (see Figure 13).

\subsection{ASK receiver}

$\mathrm{RF}$ receivers are electronic devices that separate radio signals from one another and convert specific signals into audio, video, or data formats. RF receivers use an antenna to receive transmitted radio signals and a tuner to separate a specific signal from all of the other signals that the antenna receives. Detectors or demodulators then 
extract information that was encoded before transmission. Radio techniques limit localized interference and noise; RF receivers vary in terms of performance specifications such as sensitivity, digital sampling rate, measurement resolution, operating frequency, and communication interface. Sensitivity is the minimum input signal required to produce a specified output signal having a specified signal-to-noise $(\mathrm{S} / \mathrm{N})$ ratio. Digital sampling rate is the rate at which samples can be drawn from a digital signal in kilo samples per second. Measurement resolution is the minimum digital resolution, while operating frequency is the range of received signals. Communication interface is the method used to output data to computers.

\subsection{Antenna}

Antenna is the interface between the transmission line and space. Antennas are passive devices; the power radiated cannot be greater than the power entering from the transmitter and reciprocal - the same design works for receiving systems as for transmitting systems (Roy, 2002). When speaking of gain in an antenna, gain refers to the idea that certain directions are radiated better than others. A telescopic antenna is collapsible. It is a series of small diameter tubes of 6 to 8 inches in length nested one inside the other. The antenna can be extended to its full length or retracted to a small length for storage of portability.

\subsection{Liquid Crystal Display (LCD)}

A liquid crystal display (LCD) is a thin, flat display device made up of any number of color or monochrome pixels arrayed in front of a light source or reflector as shown in Figure 9. It is often utilized in battery-powered electronic devices because it uses very small amounts of electric power.

We used LCD to view the output for our project. The LCD that we used is similar to the one in the previous figure having two rows and 16 character each one of the pixels are addressed one at a time by row and column addresses. This type of display is called a passive matrix because the pixel must retain its state between refreshes without the benefit of a steady electrical charge. As the number of pixels (and correspondingly, columns and rows) increases; this type of display becomes less feasible. Very slow response times and poor contrast are typical of passive m. The simulation of the developed system is shown in Figure 10.

\section{Future Work}

The main application of our project in future is to draw the meteorological map of Jordan as shown in Figure 14. In Jordan there are many stations, every station in every city such as Irbid, Mafraq, Salt, Safawi sends the information about the weather to main station in Amman, the capital. We can connect the information from the main station to the computer by using RS232 and we can know the weather parameters for all the country.

\section{References}

[Online] Available: http://www.amazon.com

[Online] Available: ww.ece.ndsu.nodak.edu/ glower/design/projects/2005/AV_Sensors/Final_Paper.pdf

[Online] Available: www.forum.allaboutcircuits.com

[Online] Available: www.wikipedia.com

Christopher, T. Kilian. (2005). Modern Control Technology Components and Systems. (2nd edition). Clifton Park, N.Y.: Delmar/Thomson Learning, c2006.

Gary, L. Johnson. Wind Energy Systems. [Online] Available: http://www.rpc.com

Material-handling.globalspec.com

Roy, Blake. (2002). Electronic communication system. (2nd edition). Cengage Learning India. 


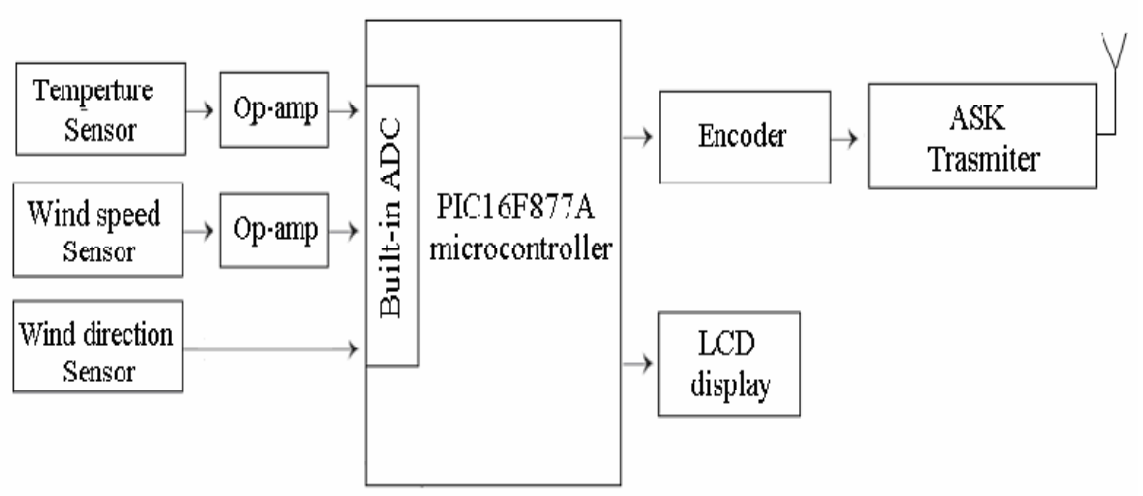

Figure 1. Block diagram of transmitting station

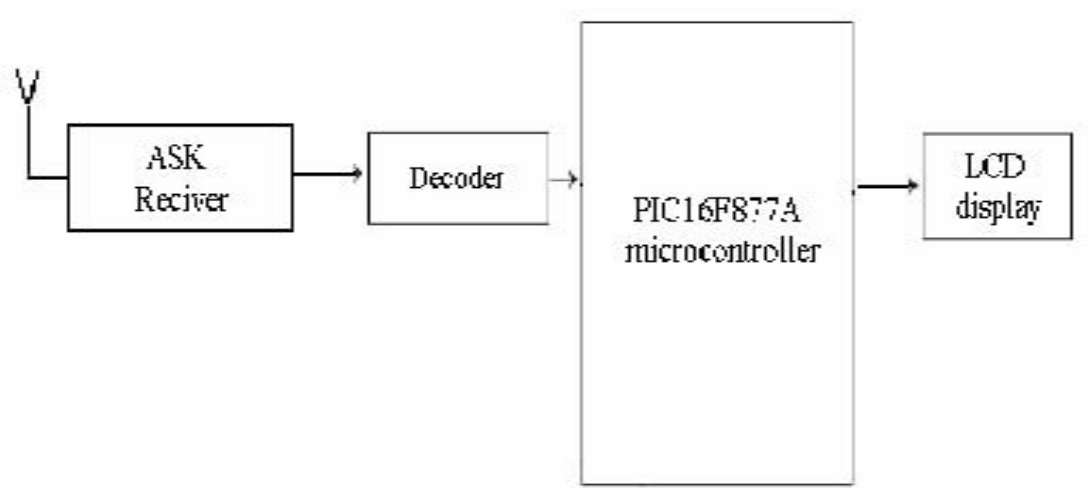

Figure 2. Block diagram of receiving station
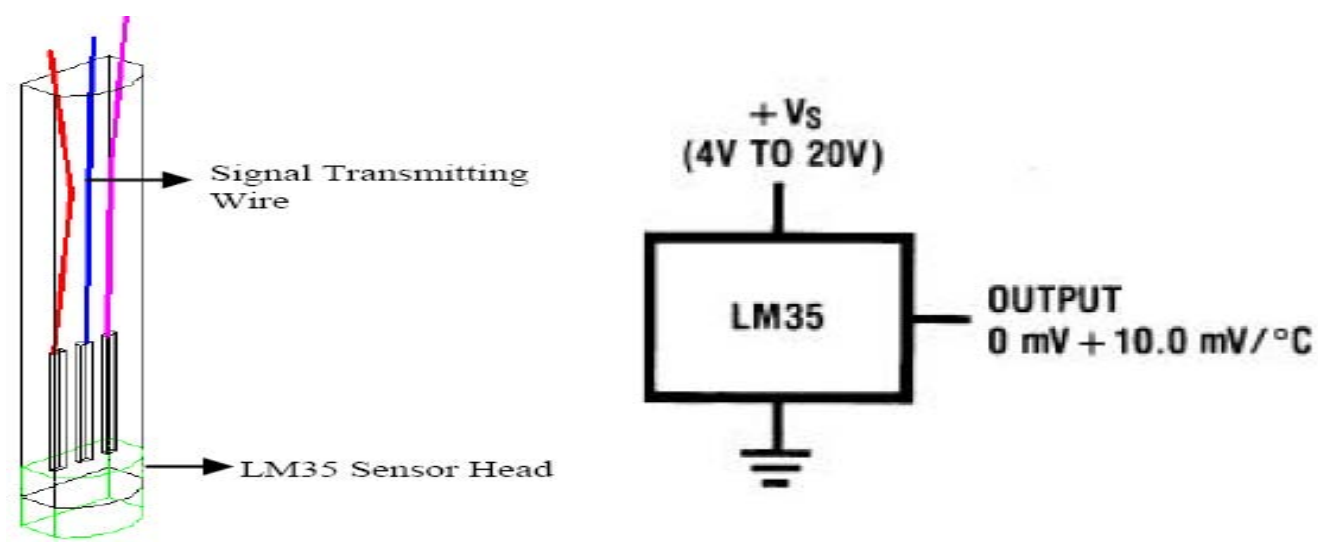

Figure 3. Temperature sensor LM35 


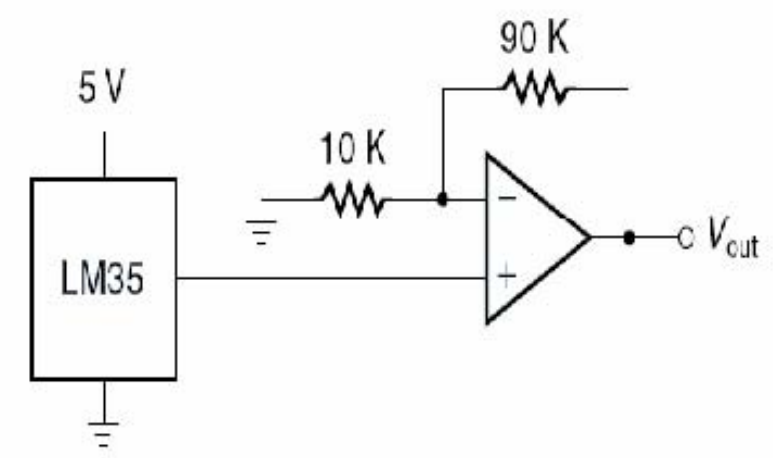

Figure 4. LM35 with TL071cp op-amp

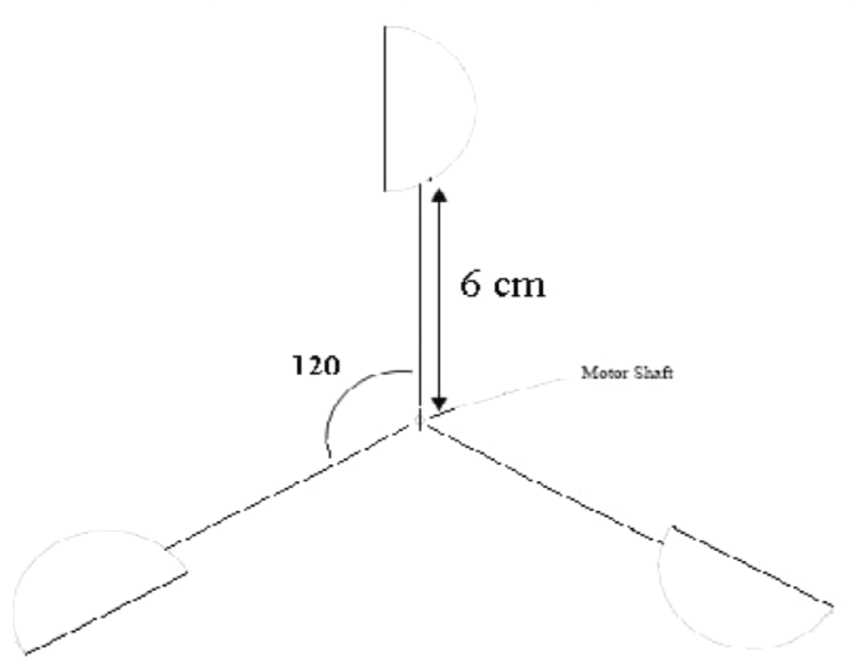

Figure 5. Cups mounted on a rotating shaft

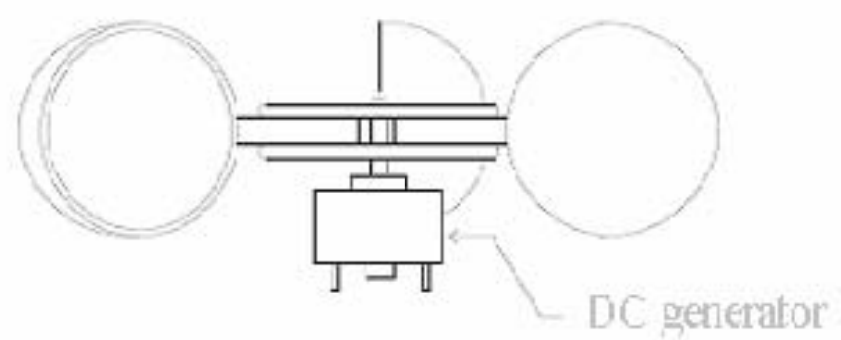

Figure 6. Cup-anemometer 


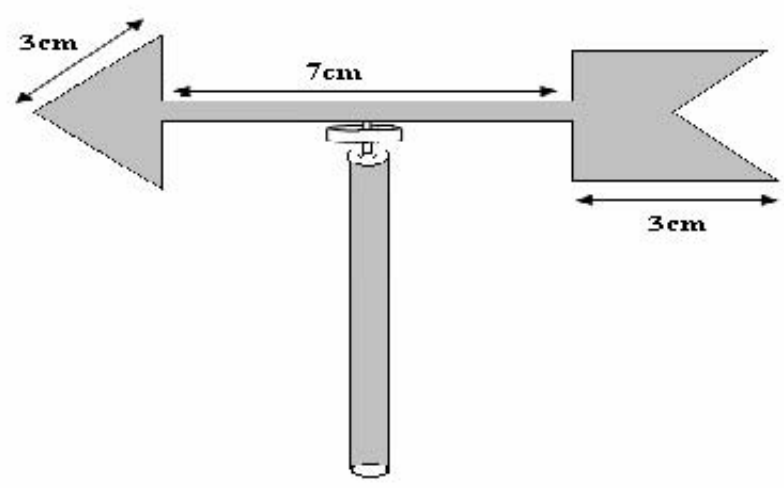

Figure 7. Wind vane

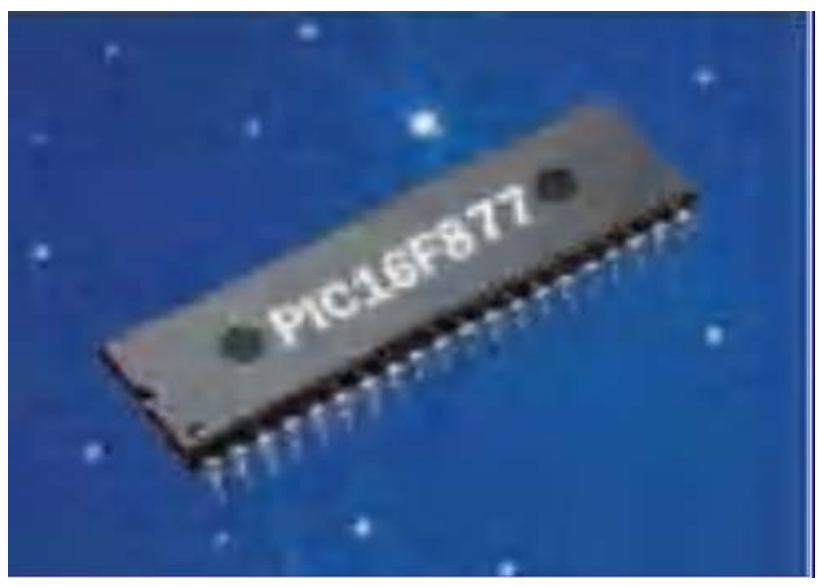

Figure 8. PIC 16F877 microcontroller

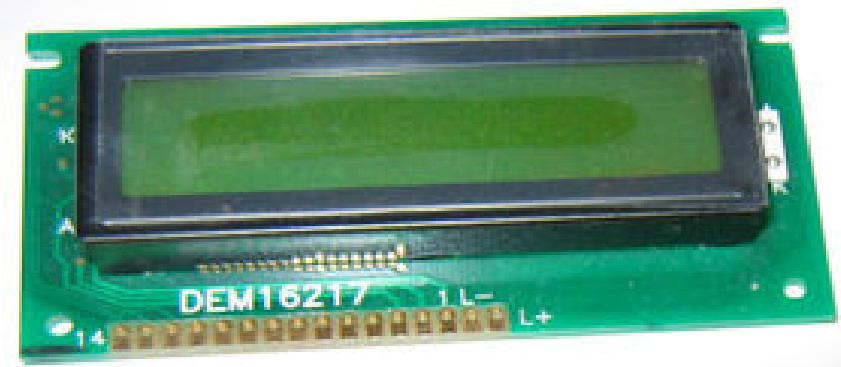

Figure 9. A general purpose alphanumeric LCD, with two lines of 16 characters 


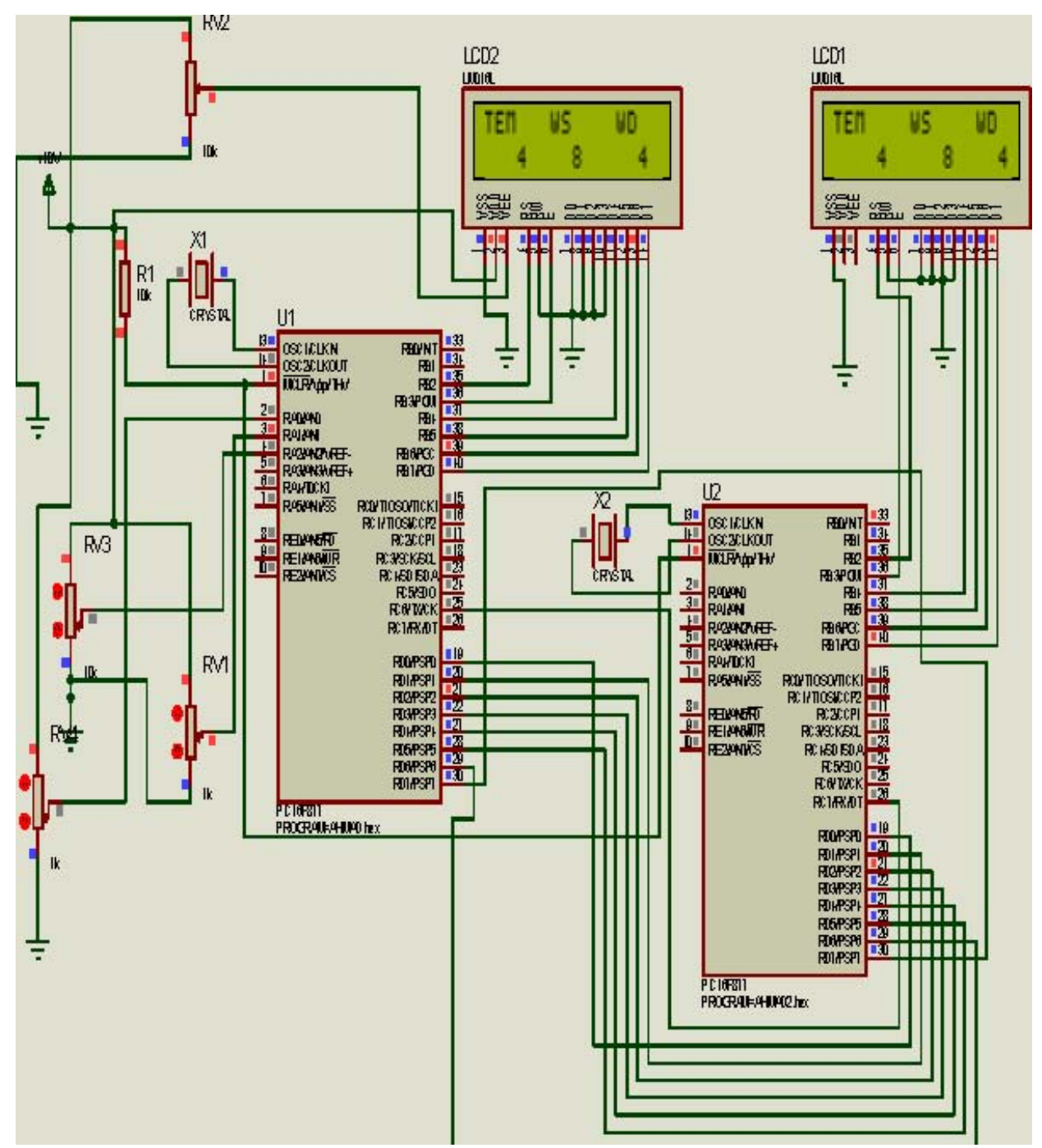

Figure 10. Simulation of the system

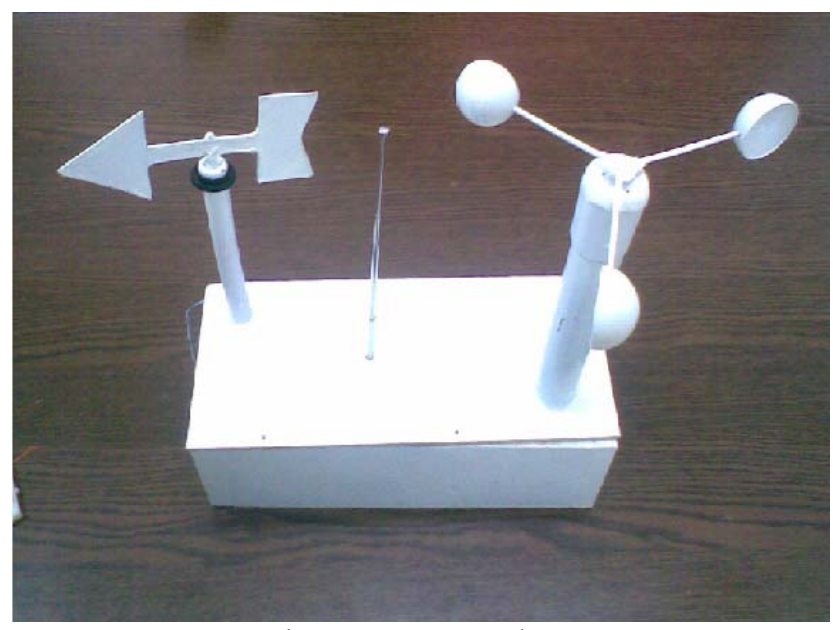

Figure 11. Sensor box 


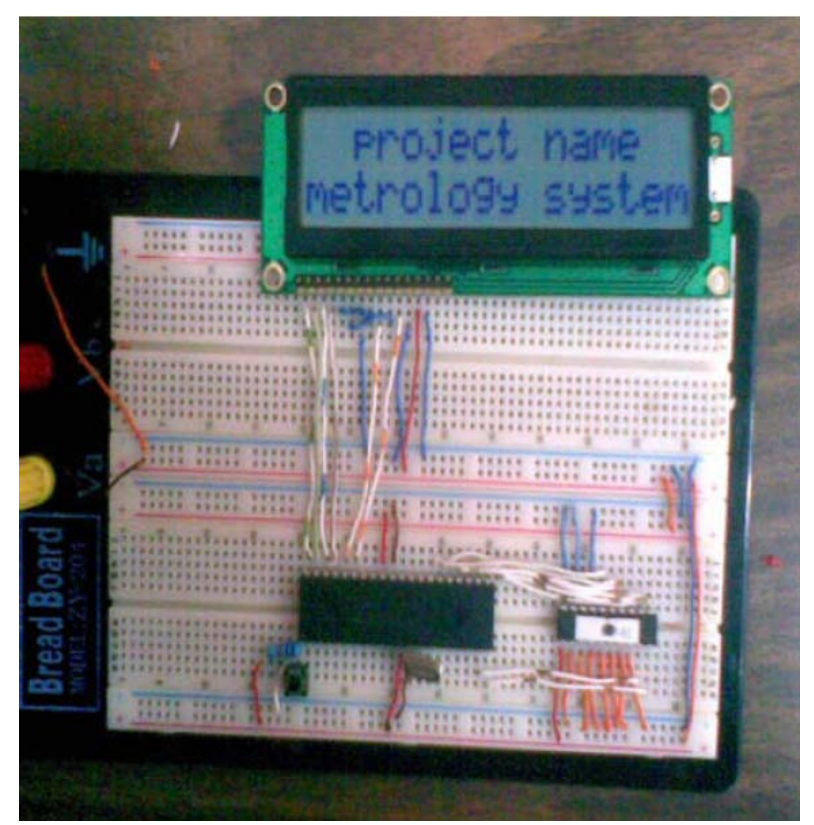

Figure 12. Transmitting station

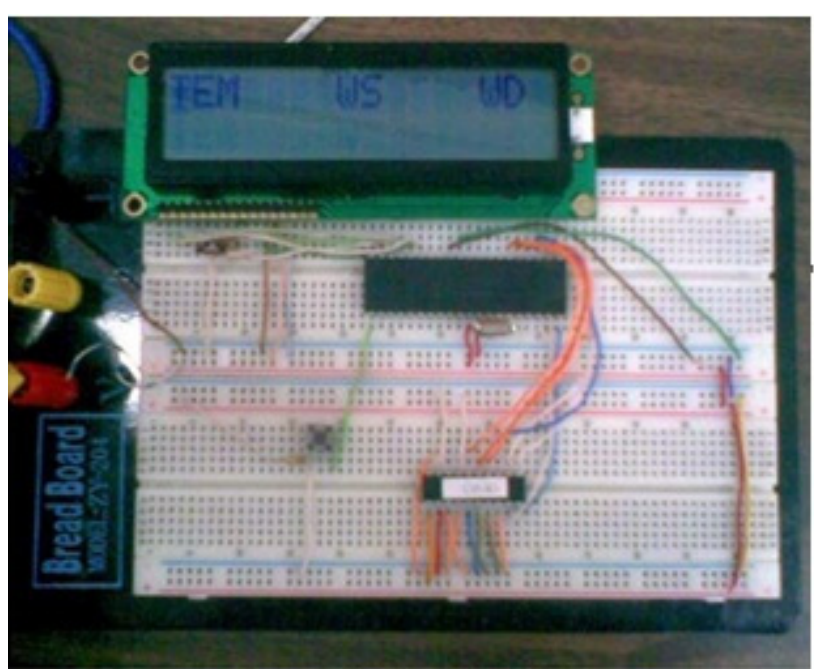

Figure 13. Receiving station 


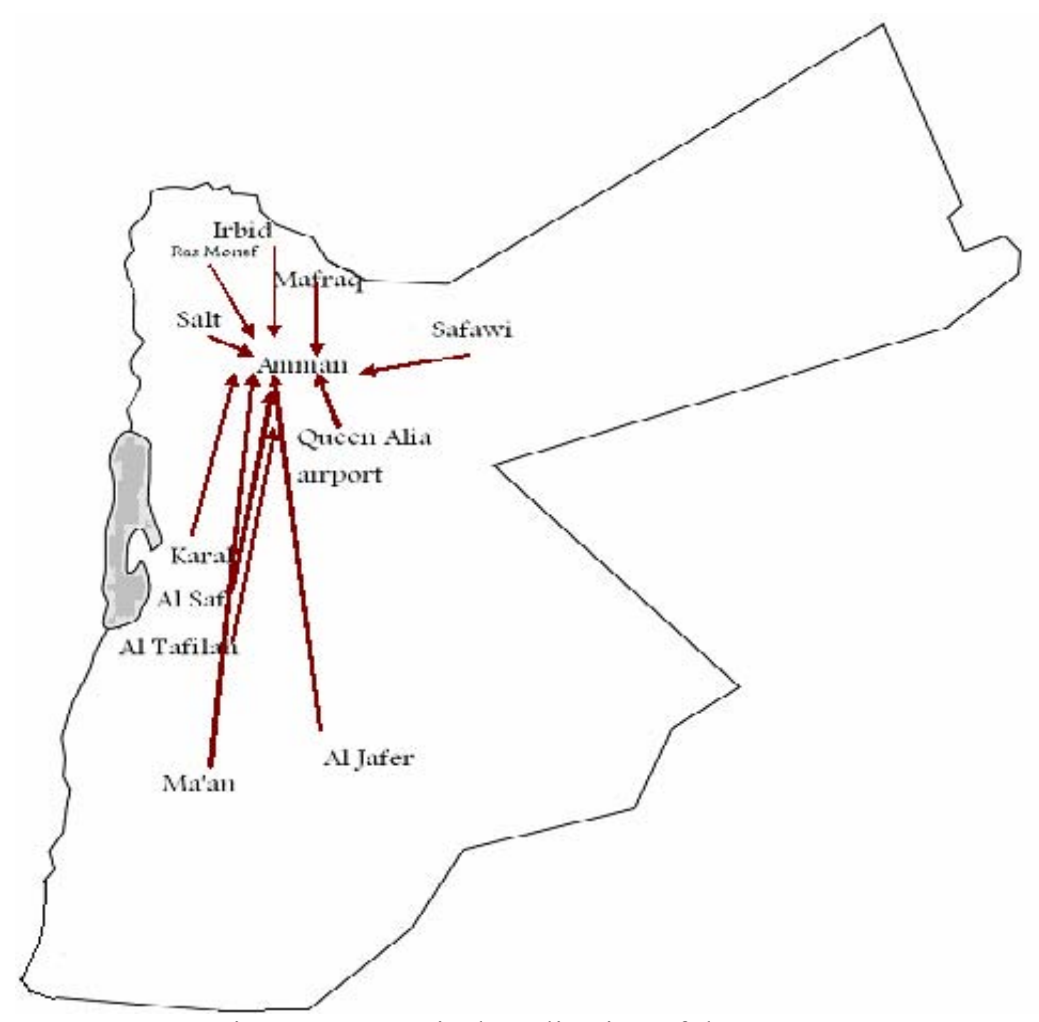

Figure 14. Practical application of the system 